\title{
Robotic-Assisted Roux-en-Y Gastric Bypass: Learning Curve Assessment Using Cumulative Sum and Literature Review
}

\author{
Roberto Bustos, MD, Alberto Mangano, MD, Federico Gheza, MD, Liaohai Chen, PhD, \\ Gabriela Aguiluz-Cornejo, MD, Antonio Gangemi, MD, FACS, Lisa Sanchez-Johnsen, PhD, \\ Chandra Hassan, MD, FACS, FRCS, and Mario Masrur, MD, FACS
}

Purpose: Robotic-assisted Roux-en-Y gastric bypass (RARYGB) is a procedure that is used with increasing frequency in the United States. Among other bariatric procedures, RARYGB is a good model for the robotic platform because it allows hand-sewn suturing and energy devices application. The aim of this study was to conduct a literature review of robotic approach in RARYGB, its learning curve using the cumulative sum (CUSUM) method, and our experience as Center of Excellence recognized by the American Society for Metabolic and Bariatric Surgery (ASMBS).

Methods: A total of 67 patients were included. Results revealed that the learning curve was achieved after case 11. Eighteen studies were included in the pooled analysis.

Results: An increase in the operative time was noted at case 46, in which a second phase was identified. A significant difference between these two phases was found only related to previous bariatric surgery. The outcomes of this series were comparable with the ones available in the literature.

Conclusions: The robotic platform is increasing its role in complex procedures such as RARYGB. The handsewn technique may offer important advantages in terms of shorter learning curve, reduced conversion rate, and lower leakage rate.

Keywords: robotic, Roux-en-Y, gastric bypass, learning curve, cumulative sum, review

\section{Introduction}

$\mathbf{S}^{\mathrm{n}}$ INCE ITS InTroduction, the da Vinci Surgical System (Intuitive Surgical, Sunnyvale, CA) has been the most widely used telemanipulator system for robot-assisted surgery. The main advantages of this system are the comfortable ergonomics for the surgeon, three-dimensional (3D) HD Vision with up to 10-fold magnification and an immersive surgical field-of-view, and wristed instrumentation with 7 degrees of freedom and software features (including motion scaling and tremor filtering). ${ }^{1}$ This technology has been applied to the field of bariatric surgery since the early stages of robotic surgery, ${ }^{2}$ and bariatric surgery is currently one of the fastest growing surgical fields. ${ }^{3}$

The laparoscopic Roux-en-Y gastric bypass (LRYGB) is one of the most challenging procedures in bariatric surgery. ${ }^{4}$ It demands high-level expertise in specific laparoscopic skills, such as accurate needle handling, intracorporeal knot tying, and bimanual tissue manipulation. Furthermore, the limitations in laparoscopic equipment (two-dimensional visualization, fulcrum effect, limited range of motion of the instruments, and surgeon fatigue caused by abdominal wall torque) make this approach even more challenging. ${ }^{5}$ As such, an increasing number of surgery centers are adopting the robotic system for the surgical treatment of obesity. ${ }^{6}$

Although the efficacy of the robotic platform in roboticassisted Roux-en-Y gastric bypass (RARYGB) has been reported, the use of this technology in bariatric surgery is controversial because of the technology-related costs. ${ }^{7}$ Some prospective studies suggest an advantage (or comparable outcomes in comparison with laparoscopy) in terms of decreased complication rate, length of hospitalization, conversion rate, and need for revisional surgery. ${ }^{8-10}$

A matched-cohort study compared RARYGB with LRYGB using the database from the 2015 Metabolic and Bariatric Surgery Accreditation and Quality Improvement Program ${ }^{11}$ (MBSAQIP). Results favored laparoscopy compared with open surgery for its reduced operative time and Illinois. 
costs, with comparable results for other perioperative outcomes. Nevertheless, the longer learning curve and dexterity that laparoscopy demands for this type of procedure should be considered. ${ }^{12,13}$

The learning curve in RARYGB has been assessed with different methods, providing heterogeneous outcomes with no clear conclusions. Despite concerns regarding a lack of agreement for a standardized method to assess the learning curve ${ }^{14}$ the cumulative sum (CUSUM) analysis is a wellestablished method to monitor the variation of a specific parameter such as the learning curve. As such, for purposes of this article, the CUSUM method was selected to compare the learning curve of RARYGB with the previous experiences reported in literature. Because of the heterogeneity regarding surgical outcomes and the need for additional research in this area, the aim of this study was to conduct a literature review of robotic approach in RARYGB, its learning curve using the CUSUM method, and our experience as Center of Excellence recognized by the American Society for Metabolic and Bariatric Surgery (ASMBS).

\section{Methods}

\section{Case series}

This is a retrospective study of a prospectively maintained database of patients who underwent RARYGB between January 2016 and December 2017 at our institution. Inclusion criteria were as follows: (1) patients who underwent RARYGB during the time period January 2016 to December 2017 and (2) patients who were 18 years of age and older. Exclusion criteria were those who did not provide informed consent. This study was approved by the Institutional Review Board at our institution (IRB protocol \#2011-1104).

The following data were retrieved from the electronic medical records: demographic information (age, sex, and race); medical information (body mass index [BMI], American Society of Anesthesiologists [ASA] score, history of previous abdominal surgeries); intraoperative information (type of surgery, associated procedure, operative time, conversion, estimated blood loss, leak test, swallow study, gastroscopy, complications, and drain usage), and postoperative information (length of stay, readmission, reoperation, morbidity, mortality, and 30-day follow-up). Data were analyzed with Microsoft Excel 2013 (Microsoft Corporation, Redmond, WA) and Stata 15 (StataCorp LLC, College Station, TX).

\section{Learning curve}

The mathematical model CUSUM was originally designed to identify if an industrial process was "under control" or "out of control" and it was first used to assess the quality control tests for ammunition production lines during World War II. ${ }^{15}$ In this original formulation, the CUSUM curve often failed to identify a reliable number of cases and it was more useful to see if in a case series, a particular event (new approach, new device, different guidelines, etc.) could alter the outcome.

For this series, the learning curve was assessed by the CUSUM. ${ }^{16}$ The portion of the curve where the maximal change in slope begins to decrease signifies the completion of the learning curve. ${ }^{17}$ The outcome used for the learning curve was the total operative time, starting from skin incision until skin closure. Other perioperative outcomes (estimated blood loss and surgical complications) were not suitable to build a CUSUM chart, because of the low value/rate in our series.

\section{Literature review}

A comprehensive literature review was performed using PubMed, Embase, and Scopus, using "robot OR robotic," "laparoscopic OR laparoscopy," "Roux-en-Y," "gastric bypass," and "learning curve" as key words and "AND" as Boolean operator. Inclusion criteria were as follows: (1) articles written in English; (2) full-text availability; (3) topic pertinent to outcomes in the RARYGB; (4) sample: adult patients, BMI ranging from $35 \mathrm{~kg} / \mathrm{m}^{2}$, with and without previous bariatric surgery. Exclusion criteria were as follows: (1) case reports; (2) topic not pertinent to outcomes in the RARYGB and its learning curve; (3) conference abstract, editorials, and other studies not showing original data.

\section{Description of surgical technique}

The adopted approach was total RARYGB with handsewn anastomosis in a single-docking manner. This technique has been previously described by our group ${ }^{18}$ and only the main steps of the procedure are reported here. Using a robotic platform, the patient is positioned in $15^{\circ}-20^{\circ}$ reverse Trendelenburg. The trocars are placed as given in Figure 1.

The dissection of the stomach starts at $\sim 5 \mathrm{~cm}$ caudal from the esophagogastric junction on the lesser curvature. A small gastric pouch is created using perigastric technique with two to three firings of the Endo GIA ${ }^{\mathrm{TM}}$ Reinforced Reload (Reinforced GIA) with Tri-Staple ${ }^{\mathrm{TM}}$ Technology (Covidien, Mansfield, MA). Roux limb of jejunum is prepared, measuring $\sim 50 \mathrm{~cm}$ distal to the ligament of Treitz, and the jejunum is divided there with Tan load linear stapler. It is then measured $140 \mathrm{~cm}$ more to construct the Roux limb. The site is identified to construct the jejunojejunostomy, and the alimentary limb is then brought up to the gastric pouch after

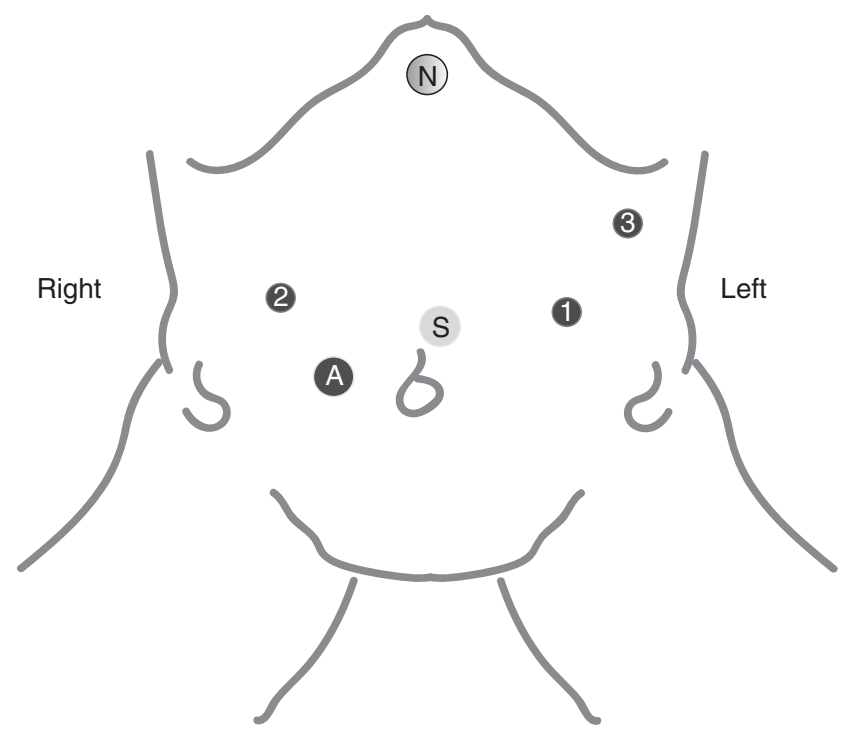

FIG. 1. Port placement. S, scope; A, assistant port; 1,2,3, robotic trocars; N, Nathanson's retractor. 
dividing the omentum. A $1.5 \mathrm{~cm}$ gastrostomy is made using monopolar hook and a hand-sewn antecolic antegastric gastrojejunostomy in two layers with PDS 3-0. The third robotic arm is used from the left side of the patient that decreases the dependence on the assistant and allows the surgeon to directly control the retraction. Finally, an intraoperative esophagogastroscopy is routinely performed and the air leak test is carried out in all cases at the end of the surgical procedure.

\section{Results}

\section{Case series}

As given in Table 1, a total of 67 patients met the eligibility criteria for inclusion in this study. The majority of the sample comprised women $(n=62)$. The average age was 44.39 years $(\mathrm{SD}=10.13), \mathrm{BMI}$ was $44.75(\mathrm{SD}=9.78)$, ASA score II, 9 patients, and ASA score III, 58 patients. A total of 46 patients had a previous abdominal surgery and 16 of those were bariatric surgeries.

Perioperative outcomes are provided in Table 2. The average operative time was $160.18 \mathrm{~min}(\mathrm{SD}=27.9 \mathrm{~min})$, average length of stay was 1.58 days $(\mathrm{SD}=0.74)$, and there were no leaks, conversions, reoperations, or mortality in this series. Morbidity was $10.4 \%$ and relevant outcomes are given in Table 2.

\section{Learning curve and CUSUM}

Figure 2 illustrates the learning curve for the total operative time. These results were compared with the previous experiences reported in literature in Table 3. The learning curve was achieved in case 11 . In the second part of the series (at case 46), an increasing trend in total operative time was noted. A significant difference between these two phases was found to be related to previous bariatric surgery (19\% vs. $37 \%, t=18.68, p \leq 0.05)$.

\section{Literature review}

Results from the literature search revealed 369 articles, of which 124 were obtained in full text. As given in Table 3, 18 studies were selected according to inclusion/exclusion criteria and included in the analysis. A total of 2,177 patients

Table 1. Demographic Patient Information

\begin{tabular}{lc}
\hline $\mathrm{N}=67$ & $\mathrm{n}(\%)$ or mean $(\mathrm{SD})$ \\
\hline Sex & \\
Female & $62(92.53 \%)$ \\
Male & $5(7.47 \%)$ \\
Age & $44.39(10.13)$ \\
BMI & $44.75(9.78)$ \\
ASA score & \\
II & $9(13.43 \%)$ \\
III & $58(86.57 \%)$ \\
Previous abdominal surgery & $46(68.66 \%)$ \\
Previous bariatric surgery & $16(23.88 \%)$ \\
Sleeve & $8(11.94 \%)$ \\
RYGB & $4(5.97 \%)$ \\
Gastric band & $4(5.97 \%)$ \\
\hline
\end{tabular}

BMI, body mass index; ASA, American Society of Anesthesiologists.
Table 2. Perioperative Outcomes

n $(\%)$ or mean $(S D)$

Overall sample

Conversion

$67(100 \%)$

0

Intraoperative complications

Estimated blood loss

Hiatal hernia repair

Drain placement

Leak

Operative time

Length of stay

Morbidity

$<30$ days postoperative complications ${ }^{\mathrm{a}}$

$>30$ days postoperative complications ${ }^{b}$

Readmissions $^{\mathrm{c}}$

Reoperation

0

$17.53(7.35)$

$5(7.46 \%)$

$23(34.33 \%)$

0

$160.18(27.9)$

$1.58(0.74)$

$7(10.4 \%)$

$1(1.5 \%)$

$2(3 \%)$

$4(6 \%)$

0

Mortality

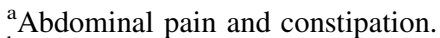

${ }^{\mathrm{b}}$ Partial internal hernia $(n=1)$, dumping $(n=1)$.

${ }^{\mathrm{c}}$ Partial obstruction improved with medical treatment $(n=1)$, postoperative oral intolerance $(n=3)$.
}

were included in the pooled analysis. A pooled analysis across all studies for which we had access to in the literature was conducted and compared with the experience of the single surgeon. A Student's $t$-test was conducted comparing the total operative time in our case series (as given in Fig. 2) with the total operative time reported in the previous literature review as reported in this study. Results revealed that the total operative time was significantly lower in our case series compared with the previous literature $(t=3.48$, Confidence interval [CI] 95\%, $p \leq 0.05$ ).

\section{Discussion}

\section{Learning curve and CUSUM}

According to our analysis, the learning curve was achieved after case 11. A visual inspection of the data allowed us to identify two phases in the learning curve. The first phase ended at case 45 . The second phase started at case 46 , where a trend to increasing total operative time was identified. After the analysis was performed comparing demographics, intraoperative and postoperative outcomes, there was only a significant difference between the two phases related to previous bariatric surgery. Results also revealed that the outcomes (total operative time, learning curve, conversion, leak, strictures, and reoperation rates) of the single surgeon were comparable with the previous literature, despite the use of different methods to assess learning curve.

\section{Literature review and comparison with this our case series}

The first RARGYB in the world was performed at our institution in 2000 and described in a published article in $2001 .^{2}$ The authors of that previous study described a series of seven patients, but the approach was hybrid because of the limitations of the robotic instruments at that time (staplers and ultrasonic scalpel were not yet available). In fact, the robotic system was only used to perform the robotic hand-sewn gastrojejunostomy.

The first fully RARYGB series was reported in the literature in 2005. Mohr et al. ${ }^{5}$ proposed a standardized technique 


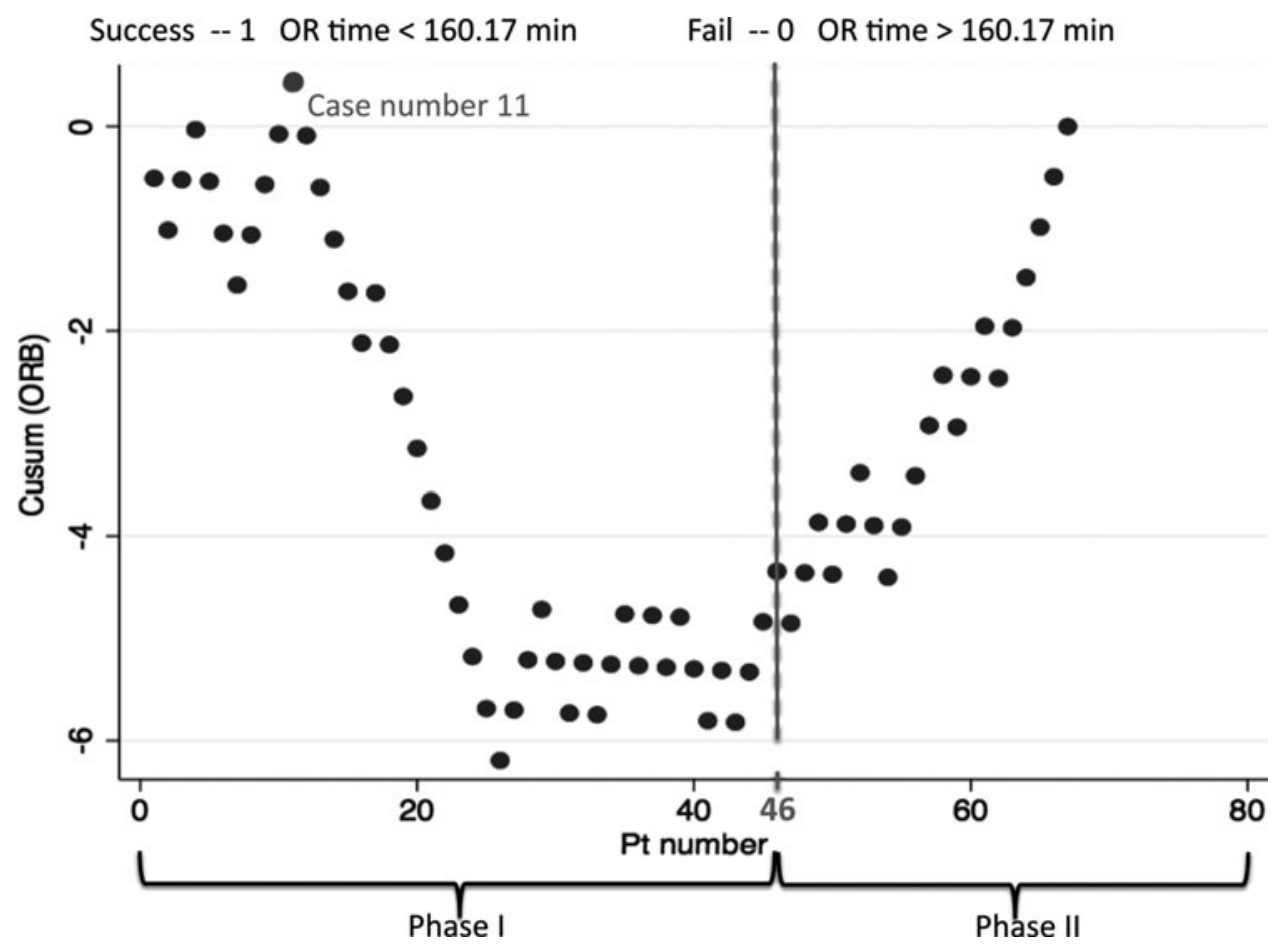

FIG. 2. Cumulative sum. Learning curve (LC) $\mathrm{C}$ was achieved after case 11 . A second phase was identified at case 46 .

for the port setting and positioning that allowed the surgery to be performed with a fully robotic method. Results from that study revealed a significantly lower operative time for the robotic group compared with the laparoscopic group (169 min vs. $208 \mathrm{~min}, p=0.03$ ).

Benizri et al. ${ }^{19}$ observed a shorter mean operative time for the robotic arm (130 min vs. $147 \mathrm{~min}, p=0.0001)$, which is in contrast to other studies. ${ }^{20-25}$ However, the results with other outcomes did not favor the RARYGB method. Surgical complications $(1 \%$ vs. $13 \%, p=0.001)$, reoperations $(1 \%$ vs $9 \%, p=0.02)$, and hospital stay (6.7 $\mathrm{SD}=1.2$ vs. $9.3 \mathrm{SD}=6.3$, $p=0.0001)$ were higher after RARYGB. Although this study was a controlled, prospective, and comparative investigation, it was not a randomized study. Additional limitations of that study included that there were different anastomosis techniques between arms and each group had its own surgeon. Moreover, there may have been possible biases related to the different anastomotic techniques performed (hand sewn vs.

Table 3. Pooled Analysis

\begin{tabular}{|c|c|c|c|c|c|c|c|c|c|}
\hline & $\mathrm{N}$ & $B M I$ & $O T$ & $L C$ & Conversion \% & Complication \% & Leak \% & Stricture \% & Reoperation \% \\
\hline Ahmad et al. ${ }^{47}$ & 172 & 47.4 & 155 & & - & - & - & 0 & 0 \\
\hline Artuso et al. ${ }^{20}$ & 41 & 52.8 & 289 & & - & - & 2.4 & - & - \\
\hline Ayloo et al. ${ }^{30}$ & 41 & 48 & 207 & & - & 2.2 & 0 & 1.1 & 1.1 \\
\hline Benizri et al..$^{19}$ & 100 & - & 130 & & 3 & 24 & 3 & & \\
\hline Bhandari et al. ${ }^{48}$ & 140 & 43.7 & 97.5 & & - & 2.9 & 0 & 1.4 & 0 \\
\hline Buchs et al. ${ }^{10}$ & 388 & 45 & 245 & 14 & 0.8 & 11.6 & 0.3 & - & 1 \\
\hline Elli et al. & 82 & 45.5 & 197.5 & & 1.2 & 2.4 & 0 & 0 & 1.2 \\
\hline Hubens et al. ${ }^{21}$ & 45 & 48.4 & 212 & & 11.1 & 6.7 & 0 & 4.4 & \\
\hline Mohr et al. ${ }^{5}$ & 21 & 44.2 & 181.7 & & - & 14 & - & - & - \\
\hline Moon et al. & 64 & 45.7 & 0 & & - & - & 7.8 & & 7.8 \\
\hline Myers et al. ${ }^{46}$ & 100 & 46.8 & 144 & & - & - & - & 6 & \\
\hline Park et al. ${ }^{22}$ & 105 & 45.5 & 169 & & - & 9.5 & 1.9 & 3.81 & 4.8 \\
\hline Renaud et al. $^{9}$ & 154 & 45.9 & 141 & 84 & 2.6 & 33.11 & 3.9 & & 9.7 \\
\hline Sanchez et al. ${ }^{8}$ & 25 & 45.5 & 130.8 & 10 & 4 & - & 4 & - & - \\
\hline Scozzari et al. ${ }^{23}$ & 110 & 40 & 247.5 & & - & 29 & 1.8 & - & 8.2 \\
\hline Smeenk et al. ${ }^{26}$ & 100 & 40 & 117 & 25 & - & 5 & 0 & 0 & - \\
\hline Snyder et al. ${ }^{24}$ & 320 & - & 193 & & - & 22.5 & 1.7 & 0.9 & - \\
\hline Starnes et al. ${ }^{49}$ & 102 & - & 147 & & - & 14.7 & 0 & 2.94 & - \\
\hline Subtotal & 2110 & 45.14 & 181.36 & - & 2.1 & 15.6 & 1.47 & 1.7 & 2.7 \\
\hline Current publication & 67 & 44.7 & 160.2 & 11 & 0 & 10.4 & 0 & 0 & 0 \\
\hline Total $N$ & 2177 & & & & & & & & \\
\hline
\end{tabular}

LC, learning curve; OT, operative time. 
stapled) and the fact that all robotic procedures were performed by the same surgeon.

In some series, it is shown that hand-sewn suturing of the anastomosis can reach leakage rates close to $0 \%{ }^{26}$ In revisional cases (including the ones with different previous bariatric procedures such as sleeve or gastric band placement), the advantages of the robotic platform with leakages are even more evident. ${ }^{27,28}$ In our case series, our results were comparable with the outcomes noted in the literature review presented in Table 3. Our results showing a $0 \%$ leak rate highlights the importance of the air leak test after the anastomosis is performed, which allows for the reinforcement of the anastomosis during the same procedure and avoidance of a reoperation in certain situations.

The few series that described the learning curve of RARYGB suggested that the surgeon needs a fewer number of cases to achieve proficiency, ranging from 10 to 30 procedures. ${ }^{8,19,26,29-31}$ However, the number needed in the laparoscopic approach varies between 75 and 100 cases. $^{12,13}$ Unfortunately, it is difficult to compare results related to operative time. The reason for this is that in some studies, the positioning and docking time were included, whereas in others, this was not considered. ${ }^{3}$ Moreover, the operative time cannot be compared between different techniques (e.g., hand-sewn vs. stapled anastomosis). Another important factor is the experience of the surgeon and of the surgical team and their stage in the learning curve for this procedure. ${ }^{3}$ In the first years of the robotic application to gastric bypass surgery, the hybrid procedure was the most commonly performed and later, there was a trend toward fully robotic surgeries. ${ }^{5}$

Buchs et al. reported the largest available series of RARYGB with long-term follow-up compared with LRYGB. ${ }^{10}$ The authors reported data on 777 consecutive minimally invasive RARYGB, with 389 cases $(50.1 \%$ ) conducted laparoscopically and 388 cases $(49.9 \%)$ conducted robotically. The reported operative time was longer in the robotic series $(245 \mathrm{~min}$ [SD = 93.6] for the robotic group vs. $215.8 \mathrm{~min}[\mathrm{SD}=69.1]$ for the laparoscopic group, $p=0.0001$ ), but short-term outcomes were better for the robotic technique. Leakage, conversion rate, reoperation, and length of stay were significantly lower in the robotic arm compared with the laparoscopic approach. Unfortunately, the retrospective nature of the study limits interpretations related to outcomes. Percent excess weight during long-term follow-up (36 months) did not favor the robotic platform (mean \% excess BMI loss: $75.2[\mathrm{SD}=22]$ for the robotic group vs. $83.6[\mathrm{SD}=19.5]$ for the laparoscopic group). The authors of that study ${ }^{10}$ reported that the lower percent excess BMI was because of technical deficiencies that should be improved (measurement of the alimentary limb and calibration of the gastrojejunal [GJ] anastomosis). Other authors hypothesized that the lower percent excess BMI is because the stoma in the hand-sewn anastomosis is generally larger than the stapled one in the laparoscopic approach and this may be a potential risk factor for less weight loss. ${ }^{32}$ In addition, the authors also noted that the length of the alimentary limb might be shorter in the robotic group than in the laparoscopic group. ${ }^{10}$ Another study hypothesized that this may be because the $3 \mathrm{D}$ vision could make the measurement of the alimentary limb more difficult. ${ }^{31}$ However, in some series, long-term weight loss did not revealed significant differences between the aforementioned approaches. ${ }^{22}$

In the literature review by Toro et al. on the role of the robotic platform in different types of procedures that involve the foregut and bariatric surgery, the authors concluded that RARYGB was safe and feasible, and suggested that this approach may shorten the learning curve. ${ }^{33}$ The authors also noted that the key advantage of the robotic system is the improved ergonomics, which is a particular benefit when working with patients who are obese. This improvement may help to prevent the situation of the surgeon becoming fatigued because of the torque required to work with such heavy and thick abdominal walls. ${ }^{33}$

Jung et al. published the latest systematic review comparing robotic and laparoscopic RARYGB. ${ }^{3}$ The review included 14 studies, of which only one was a prospective and randomized study. ${ }^{8}$ Results revealed multiple discrepancies in the leak rate. The authors stated that this is mainly because of differences related to the anastomotic technique. During the robotic procedures with hand-sewn gastrojejunostomy, the reported leak rate in the literature ranges between $0 \%$ and $3 \% .^{10,19,26,34-37}$ The vast majority of leaks in RARYGB occurred when the anastomoses were performed with stapler. This is also true with the outcomes reported by Moon et al., ${ }^{38}$ where the laparoscopic group had a lower leak rate $(7.8 \%$ for laparoscopy vs. $0.5 \%$ for robotic-assisted procedures, $p \leq 0.01)$. This could be explained by the fact that stapling of the gastric pouch in robotic procedures was performed by the bedside assistant (usually less experienced compared with a surgeon), whereas all stapling in the laparoscopic surgeries were conducted by a surgeon.

The only randomized study comparing LRYGB and RARYGB involved 50 patients. ${ }^{8}$ The procedures were performed by a new laparoscopic fellow with no previous experience in gastric bypass but with experience in other complex laparoscopic procedures. Results from this study revealed that there were significant differences in the mean operative time between the surgeries (RARYGB: $130.8 \mathrm{~min}$. vs. LRYGB $149.4 \mathrm{~min}, p=0.02$ ) and a greater difference in procedure time when patients had BMI $>43 \mathrm{~kg} / \mathrm{m}^{2}$ in a post hoc analysis (RARYGB: $123.5 \mathrm{~min}$ vs. LRYGB: $153.2 \mathrm{~min}$, $p=0.009)$. There were no significant differences between age, gender, comorbidities, complication rates, and length of stay. ${ }^{8}$

Despite the heterogeneity in outcomes reported in the literature, our morbidity rates related to leak rates and strictures are comparable with previous studies. In our study, there were zero leaks and strictures (and consequently no need for reoperations). One reason for this may be related to the extensive training in anastomosis construction in the robotic skills laboratory by the surgeon, before operating with patients.

The robotic platform role in revisional surgery is controversial. It is known that reoperation can increase the risk of bleeding and anastomotic complications. ${ }^{39}$ Moreover, the robotic platform can increase the chances of conversion and it may be very challenging, even for the experienced bariatric surgeon. ${ }^{28}$ Some authors consider adhesions, scarring, and fibrosis in the surgical field as a contraindication for the minimally invasive approach. ${ }^{40}$ In addition, other authors believe that robotic platform dramatically facilitates the safety of these procedures and minimizes the need to convert to an open procedure. ${ }^{41}$

The largest sample of RARYGB as revisional procedure included 32 patients. ${ }^{28}$ In this study, revisional RARYGB was indicated after weight loss failure, weight regain, or complications of the primary bariatric surgery. There were no conversions, postoperative leaks, hemorrhages, strictures, or 
reoperations within 30 days, and no mortalities reported in the entire series. The authors utilized a hand-sewn technique for this particular scenario. After the anastomosis was performed, an intraoperative endoscopic check was routinely conducted, which identified two leaks that were repaired in the same surgical intervention, using PDS 3-0 suture. ${ }^{28}$

Renaud et al. conducted a prospective study of 154 RARYGB patients. ${ }^{9}$ The authors examined the role of the surgeons' experience, bedside first assistant, and patient factors on operative time and postoperative complications. Results from a multivariate analysis revealed that case rank and first assistant level were the only independent factors related to total operative time. A learning curve was performed with CUSUM analysis, and the authors included operative time, in addition to conversion, morbidity, hospital stay, and rehospitalization. The learning curve according to this method was achieved after case 84, and the authors concluded that the learning curve between RARYGB and LRYGB were comparable. ${ }^{12,13}$

Accredited centers around the United States (Metabolic Bariatric Surgery and Quality Improvement Program and Centers for Medicare and Medicaid Services) have better outcomes compared with nonaccredited centers, suggesting that a formal training program is recommended to obtain better results. $^{42}$ In 2014, our group published its 5-year experience related to RARGYB (a retrospective review of 118 RARYGB hybrid and 82 fully robotic cases) from our bariatric surgery program accredited by the ASMBS-QIP. ${ }^{18}$ Results revealed no leaks, hemorrhage requiring transfusion, or gastrojejunostomy strictures in the entire series, whereas the previously reported complication rate ranged from $0 \%$ to $7 \%$ in GB strictures, from $0 \%$ to $2 \%$ in GJ leaks, and from $0 \%$ to $9 \%$ for anastomotic bleeding. ${ }^{29,43-47}$ Those data suggest that the technique standardization and the experience of the team with the robotic platform may reduce negative outcomes. ${ }^{18}$ Our bariatric center strongly supports performing this kind of procedure only in a controlled and accredited environment.

There are several limitations to this study. First, the retrospective nature of this study limits inferences about the causal nature of the relationships discussed here. Second, there was a single surgeon performing the procedure. However, the distinctiveness was that the surgeon also had previous experience in laparoscopic and robotic surgical procedures other than RARYGB. Third, the technique for RARGYB is not standardized and the method to determine a learning curve is different in each study in literature, resulting in a decreased value of the pooled analysis. Finally, this was a relatively small sample size that limits its statistical power.

\section{Conclusion}

The robotic platform is increasing its role in complex procedures such as RARYGB. ${ }^{48,49}$ The hand-sewn technique can offer important advantages in terms of shorter learning curve, reduced conversion rate, and lower leakage rate. However, additional research in this area is needed because of the heterogeneity of the studies that were analyzed. The strength of this study is that it is a single surgeon experience at an institution with a standardized technique for conducting RARYGB. Overall, we believe that perioperative outcomes are highly influenced by the surgeon's previous experience (other robotic procedures or open/laparoscopic bypass) and by skills of the table assistant. Robotic surgery requires a specific training for each member of the team and not only for the surgeon at the console.

\section{Acknowledgment}

This research was not conducted with any specific grants from funding agencies in the public, commercial, or not-forprofit sectors.

\section{Author Disclosure Statement}

No competing financial interests exist.

\section{References}

1. Hagen ME, Jung MK, Ris F, et al. Early clinical experience with the da Vinci Xi Surgical System in general surgery. J Robotic Surg 2017;11:347-353.

2. Horgan S, Vanuno D. Robots in Laparoscopic Surgery. J Laparoendosc Adv Surg Tech 2001;11:415-419.

3. Jung MK, Hagen ME, Buchs NC, Buehler LH, Morel P. Robotic bariatric surgery: a general review of the current status. Int J Med Robotics Comput Assisted Surg 2017;13: e1834-n/a.

4. Stefanidis D, Bailey SB, Kuwada T, Simms C, Gersin K. Robotic gastric bypass may lead to fewer complications compared with laparoscopy. Surg Endosc 2018;32:610616.

5. Mohr CJ, Nadzam GS, Curet MJ. Totally robotic Roux-enY gastric bypass. Arch Surg 2005;140:779-786.

6. Parisi A, Reim D, Borghi F, et al. Minimally invasive surgery for gastric cancer: a comparison between robotic, laparoscopic and open surgery. World J Gastroenterol 2017;23:2376-2384.

7. Higgins RM, Frelich MJ, Bosler ME, Gould JC. Cost analysis of robotic versus laparoscopic general surgery procedures. Surg Endosc 2017;31:185-192.

8. Sanchez BR, Mohr CJ, Morton JM, Safadi BY, Alami RS, Curet MJ. Comparison of totally robotic laparoscopic Rouxen-Y gastric bypass and traditional laparoscopic Roux-en-Y gastric bypass. Surg Obes Relat Dis 2005;1:549-554.

9. Renaud M, Reibel N, Zarnegar R, et al. Multifactorial analysis of the learning curve for totally robotic Roux-en-Y gastric bypass for morbid obesity. Obes Surg 2013;23: 1753-1760.

10. Buchs NC, Morel P, Azagury DE, et al. Laparoscopic versus robotic Roux-en-Y gastric bypass: lessons and longterm follow-up learned from a large prospective monocentric study. Obes Surg 2014;24:2031-2039.

11. Sharma G, Strong AT, Tu C, Brethauer SA, Schauer PR, Aminian A. Robotic platform for gastric bypass is associated with more resource utilization: an analysis of MBSAQIP dataset. Surg Obes Relat Dis 2018;14:304-310.

12. Oliak D, Ballantyne GH, Weber P, Wasielewski A, Davies RJ, Schmidt HJ. Laparoscopic Roux-en-Y gastric bypass: defining the learning curve. Surg Endosc 2003;17:405-408.

13. Shikora SA, Kim JJ, Tarnoff ME, Raskin E, Shore R. Laparoscopic Roux-en-Y gastric bypass: results and learning curve of a high-volume academic program. Arch Surg 2005; 140:362-367.

14. Van Rij AM, McDonald JR, Pettigrew RA, Putterill MJ, Reddy CK, Wright JJ. Cusum as an aid to early assessment of the surgical trainee. Br J Surg 1995;82:1500-1503. 
15. Bolsin S, Colson M. The use of the Cusum technique in the assessment of trainee competence in new procedures. Int $\mathbf{J}$ Qual Health Care 2000;12:433-438.

16. Neuburger J, Walker K, Sherlaw-Johnson C, van der Meulen J, Cromwell DA. Comparison of control charts for monitoring clinical performance using binary data. BMJ Qual Saf 2017;26:919-928.

17. Young A, Miller JP, Azarow K. Establishing learning curves for surgical residents using cumulative summation (CUSUM) Analysis. Curr Surg 2005;62:330-334.

18. Bindal V, Gonzalez-Heredia R, Masrur M, Elli EF. Technique evolution, learning curve, and outcomes of 200 robot-assisted gastric bypass procedures: a 5-year experience. Obes Surg 2015;25:997-1002.

19. Benizri EI, Renaud M, Reibel N, et al. Perioperative outcomes after totally robotic gastric bypass: a prospective nonrandomized controlled study. Am J Surg 2013;206:145151.

20. Artuso D, Wayne M, Grossi R. Use of robotics during laparoscopic gastric bypass for morbid obesity. JSLS 2005; 9:266-268.

21. Hubens G, Balliu L, Ruppert M, Gypen B, Van Tu T, Vaneerdeweg W. Roux-en-Y gastric bypass procedure performed with the da Vinci robot system: is it worth it? Surg Endosc 2008;22:1690-1696.

22. Park CW, Lam ECF, Walsh TM, et al. Robotic-assisted Roux-en-Y gastric bypass performed in a community hospital setting: the future of bariatric surgery? Surg Endosc 2011;25:3312.

23. Scozzari G, Rebecchi F, Millo P, Rocchietto S, Allieta R, Morino M. Robot-assisted gastrojejunal anastomosis does not improve the results of the laparoscopic Roux-en-Y gastric bypass. Surg Endosc 2011;25:597-603.

24. Snyder BE, Wilson T, Leong BY, Klein C, Wilson EB. Robotic-Assisted Roux-en-Y Gastric Bypass: minimizing Morbidity and Mortality. Obes Surg 2010;20:265-270.

25. Hagen ME, Pugin F, Chassot G, et al. Reducing cost of surgery by avoiding complications: the model of robotic Roux-en-Y gastric bypass. Obes Surg 2012;22:52-61.

26. Smeenk RM, van 't Hof G, Elsten E, Feskens PG. The results of 100 robotic versus 100 laparoscopic gastric bypass procedures: a single high volume centre experience. Obes Surg 2016;26:1266-1273.

27. Ayloo SM, Choudhury N. Robotic revisional bariatric surgery: single-surgeon case series. Int J Med Robot 2015; 11:284-289.

28. Bindal V, Gonzalez-Heredia R, Elli EF. Outcomes of Robot-Assisted Roux-en-Y gastric bypass as a reoperative bariatric procedure. Obes Surg 2015;25:1810-1815.

29. Yu SC, Clapp BL, Lee MJ, Albrecht WC, Scarborough TK, Wilson EB. Robotic assistance provides excellent outcomes during the learning curve for laparoscopic Roux-en-Y gastric bypass: results from 100 robotic-assisted gastric bypasses. Am J Surg 2006;192:746-749.

30. Ayloo SM, Addeo P, Shah G, Sbrana F, Giulianotti PC. Robot-assisted hybrid laparoscopic Roux-en-Y gastric bypass: surgical technique and early outcomes. J Laparoendosc Adv Surg Tech Part A 2010;20:847-850.

31. Buchs NC, Pugin F, Bucher P, et al. Learning curve for robot-assisted Roux-en-Y gastric bypass. Surg Endosc 2012;26:1116-1121.

32. Abu Dayyeh BK, Lautz DB, Thompson CC. Gastrojejunal Stoma Diameter Predicts Weight Regain after Roux-en-Y Gastric Bypass. Clin Gastroenterol Hepatol 2011;9:228-233.
33. Toro JP, Lin E, Patel AD. Review of robotics in foregut and bariatric surgery. Surg Endosc 2015;29:1-8.

34. Moser F, Horgan S. Robotically assisted bariatric surgery. Am J Surg 2004;188(4A Suppl):38s-44s.

35. Ramos AC, Domene CE, Volpe P, et al. Resultados iniciais da primeira série de casos brasileira de cirurgia bariátrica totalmente robótica. ABCD Arq Bras Cir Dig (São Paulo) 2013;26:2-7.

36. Ayloo S, Roh Y, Choudhury N. Laparoscopic, hybrid, and totally robotic Roux-en-Y gastric bypass. J Robotic Surg 2016;10:41-47.

37. Stefanidis D, Bailey SB, Kuwada T, Simms C, Gersin K. Robotic gastric bypass may lead to fewer complications compared with laparoscopy. Surg Endosc 2017.

38. Moon RC, Gutierrez JC, Royall NA, Teixeira AF, Jawad MA. Robotic Roux-en-Y Gastric Bypass, is it Safer than Laparoscopic Bypass? Obes Surg 2016;26:1016-1020.

39. Hallowell PT, Stellato TA, Yao DA, Robinson A, Schuster MM, Graf KN. Should bariatric revisional surgery be avoided secondary to increased morbidity and mortality? Am J Surg 2009;197:391-396.

40. Chen RN, Moore RG, Cadeddu JA, et al. Laparoscopic renal surgery in patients at high risk for intra-abdominal or retroperitoneal scarring. J Endourol 1998;12:143-147.

41. Kim K, Hagen ME, Buffington C. Robotics in Advanced Gastrointestinal Surgery: the Bariatric Experience. Cancer J 2013;19:177-182.

42. Azagury D, Morton JM. Bariatric Surgery Outcomes in US Accredited vs Non-Accredited Centers: a Systematic Review. J Am Coll Surg 2016;223:469-477.

43. Fourman MM, Saber AA. Robotic bariatric surgery: a systematic review. Surg Obes Relat Dis 2012;8:483-488.

44. Cirocchi R, Boselli C, Santoro A, et al. Current status of robotic bariatric surgery: a systematic review. BMC Surg 2013;13:53.

45. Deng JY, Lourie DJ. 100 robotic-assisted laparoscopic gastric bypasses at a community hospital. Am Surg 2008; 74:1022-1025.

46. Myers SR, McGuirl J, Wang J. Robot-assisted versus laparoscopic gastric bypass: comparison of short-term outcomes. Obes Surg 2013;23:467-473.

47. Tieu K, Allison N, Snyder B, Wilson T, Toder M, Wilson E. Robotic-assisted Roux-en-Y gastric bypass: update from 2 high-volume centers. Surg Obes Relat Dis 2013;9:284-288.

48. Bhandari M, Mathur W, Mishra AK, Chandorkar D. Robotic Roux-en-Y gastric bypass: Our center's technique with short-term experience. J Min Access Surg 2017;13: 96-102.

49. Starnes CC, Gochnour DC, Hall B, Wilson EB, Snyder BE. The economy of motion of the totally robotic gastric bypass: technique, learning curve, and outcomes of a fellowshiptrained, robotic bariatric surgeon. J Laparoendosc Adv Surg Tech A 2015;25:411-418.

Address correspondence to: Roberto Bustos, MD

Division of General, Minimally Invasive and Robotic Surgery

Department of Surgery

University of Illinois at Chicago 840 S. Wood Street, Suite 435E (MC 958)

Chicago, IL 60612

E-mail: bustos@uic.edu 